\title{
Analysis of Partial FFT Demodulation for Doppler Distorted OFDM Signals
}

\author{
Srinivas Yerramalli ${ }^{1}$, Milica Stojanovic ${ }^{2}$, and Urbashi Mitra ${ }^{1}$ \\ ${ }^{1}$ University of Southern California, Email: $\{$ srinivas.yerramalli,ubli $\} @$ usc.edu \\ ${ }^{2}$ Northeastern University, Email: millitsa@mit.edu
}

\begin{abstract}
Accurate time scale compensation is a problem of significance for underwater acoustic channels. Using our proposed partial FFT Demodulation technique, we consider two algorithms for fast and accurate data detection over time scale distorted channels. We present a theoretical analysis to characterize the performance of these algorithms and determine the optimal values of their key parameters. Our analysis supports the observed performance trends and validates the performance gains of partial FFT demodulation.
\end{abstract}

\section{INTRODUCTION}

High speed communications over an underwater acoustic (UWA) channel have been considered challenging due to the low speed of sound $(\mathrm{c}=1500 \mathrm{~m} / \mathrm{sec})$, limited bandwidth and time-varying multipath [1]. Acoustic propagation is best supported at low frequencies where the available bandwidth is comparable to the operating frequency and hence UWA systems are wideband by nature. Orthogonal Frequency Division Multiplexing (OFDM) is now being strongly considered as the modulation of choice for UWA communications [2]-[4]. Interest in OFDM stems from the fact that it decomposes a static frequency selective channel into several flat channels, enabling low complexity one-tap equalization and symbol-bysymbol detection at the receiver. However, carrier orthogonality in wideband OFDM systems such as UWA communications is highly sensitive to motion induced distortion, wherein the Doppler causes time scaling of the signal, destroying orthogonality and resulting in inter-carrier interference (ICI).

In this paper, we consider a point to point UWA communication link with motion induced Doppler distortion. In UWA OFDM systems, the time scaling results in a different frequency offset for each subcarrier and thus significant ICI [3], [5], [6]. Several approaches have been proposed in the literature to demonstrate the viability of OFDM systems in UWA channels with time scaling. For mobile underwater transceivers, the time scaling parameter $(\mathrm{a}=\mathrm{v} / \mathrm{c}$, the ratio of the relative transmitter/receiver velocity to the speed of sound) is typically on the order of $10^{-3}$ [3], [7]. The received signal is first resampled to compensate for the time scaling. However, errors in resampling or slowly drifting transmit/receieve elements in stationary environments can result in significant residual time scaling distortion on the order of $a=10^{-4}$.

The case of very small residual time scales, i.e., $(a \approx$ $10^{-5}$ ) has been treated in [8]. By modeling the distortion as a progressively increasing phase shift across subcarriers, a decision directed block adaptive processing algorithm has been proposed. A second proposed method employs partial FFT demodulation in conjuction with differentially coherent detection to eliminate the need to estimate the time scale [9]. Adaptive equalizers which does not consider the signal structure induced by the time scale are proposed in [10], [11]. However, for larger time scale estimation errors, the distortion cannot be modeled as a phase rotation of the transmitted symbols without a severe loss in performance and the use of generic equalizers ignores the signal structure and could significantly increase the computational cost.

In our earlier work [4], [6], we proposed a new technique called partial FFT demodulation to provide a high performance detection algorithm for time scale distorted OFDM systems at moderate complexity. In the proposed scheme, the OFDM symbol duration is divided into several intervals and the time-varying channel in each such interval is modeled as a piecewise constant using a midpoint approximation. An FFT is performed by considering only the data in each partial interval instead of a single full interval FFT. Weighted combining of the partial FFT outputs leads to significantly reduced distortion. Accurately determining the weights to combine the partial FFT outputs from the distortion model is then key to reducing the ICI. In [6], we proposed two algorithms to compute the combiner weights: recursive weight estimation and model based weight estimation.

In this paper, we present an analysis to characterize and quantify the performance of the two proposed algorithms in [6] as a function of key parameters of interest. We first determine the optimal combiner weights in several scenarios. Exploiting the idea that time scaling can be approximated as a local carrier frequency offset (CFO) problem for each subcarrier, the analysis is presented for an OFDM signal with CFO and the results are then interpreted in the context of OFDM signal with time scaling distortion. We first show that using model based weight estimation monotonically increase the performance of data detection with increasing number of intervals. Next, by adapting an analysis technique proposed for blind and data aided detection in CDMA systems [12], [13], we derive the performance of the recursive estimation algorithm. Evaluating the obtained expressions shows our analysis to be in strong agreement with the numerical simulations in [6].

This paper is organized as follows. Section II presents the OFDM signal model and briefly illustrates the concept of partial FFT demodulation presented in [4]. The optimal combiner 
weights for the several practical scenarios are determined in Section III and Section IV presents an analysis of both the algorithms. The paper concludes with remarks in Section V.

\section{Signal Model And Partial FFt Demodulation}

\section{A. Signal Model}

We recall partial FFT demodulation as presented in [4], [6] and is briefly illustrated below. The transmitted OFDM signal in passband is given by

$$
s(t)=\sqrt{\frac{1}{T}} R e\left\{\sum_{l=1}^{K} d_{l} e^{j 2 \pi f_{l} t}\right\}, t \in\left[-T_{g}, T\right]
$$

where the vector $\mathbf{d}=\left[d_{1}, d_{2}, \cdots, d_{K}\right]$ of data symbols is modulated onto $K$ OFDM subcarriers and $T, T_{g}$ denote the duration of the OFDM symbol and the cyclic prefix, respectively. The $k^{t h}$ subcarrier frequency is at $f_{k}=f_{0}+(k-1) \Delta f$ and the signaling bandwidth is then $B=K \Delta f$. The time varying channel is modeled as

$$
h(\tau, t)=\sum_{p=1}^{P} h_{p}(t) \delta\left(\tau-\tau_{p}(t)\right),
$$

where $h_{p}(t)$ and $\tau_{p}(t)$ are the time-varying gain and delay of the $p^{t h}$ path. In this paper, we consider the following cases.

1) Time invariant channels: $h_{p}(t)=h_{p}$ and $\tau_{p}(t)=\tau_{p}$.

2) Carrier Frequency Offset: $h_{p}(t)=h_{p} e^{j 2 \pi f_{d} t}$ and $\tau_{p}(t)=$ $\tau_{p}$, where $f_{d}$ is the frequency offset.

3) Wideband channels with time scale: $h_{p}(t)=h_{p}$ and $\tau_{p}(t)=\tau_{p}-a t$, where $a$ is the time scaling parameter.

The partial FFT outputs for the given time-varying channel are approximated as

$$
\begin{aligned}
y_{k}(m) & \approx \sum_{p=1}^{P} \sum_{l=1}^{K} d_{l} h_{p}(m) e^{-j 2 \pi f_{l} \tau_{p}(m)} I_{l-k}(m)+n_{k}(m) \\
& =\sum_{l=1}^{K} d_{l} H_{l}(m) I_{l-k}(m)+n_{k}(m),
\end{aligned}
$$

where $h_{p}(m)$ and $\tau_{p}(m)$ are the relevant mid-point values of the path gains and delays in the interval $[(m-$ 1) $T / M, m T / M]$. The effective channel gain as seen by the $k^{t h}$ subcarrier in the $m^{t h}$ partial interval is $H_{k}(m)=$ $\sum_{p=1}^{P} h_{p}(m) e^{-j 2 \pi f_{k} \tau_{p}(m)}$, and the function $I_{i}(m)$ captures the effect of partial integration:

$$
I_{i}(m)=\frac{e^{j 2 \pi i \frac{2 m-1}{2 M}}}{M} \operatorname{sinc}\left(\frac{\pi i}{M}\right),|i| \leq K-1 .
$$

Note that $\sum_{m} I_{i}(m)=\delta_{i}$ and $I_{0}(m)=1 / M, \forall m=$ $1,2, \ldots, M$. Let us define $\mathbf{v}_{k}=\left[I_{k}(1), I_{k}(2), \cdots, I_{k}(M)\right]^{T}$ as the vector containing the partial interval integration coefficients, $\mathbf{H}_{k}=\operatorname{diag}\left[H_{k}(1), H_{k}(2), \cdots, H_{k}(M)\right]$ as the diagonal matrix containing the approximated frequency response and $\mathbf{y}_{k}=\left[y_{k}(1), y_{k}(2), \cdots, y_{k}(M)\right]^{T}$ as the vector containing the partial FFT outputs for the $k^{t h}$ subcarrier. In vector form, the partial FFT outputs for the $k^{t h}$ subcarrier can then be written as

$$
\mathbf{y}_{k}=\sum_{l=1}^{K} d_{l} \mathbf{H}_{l} \mathbf{v}_{l-k}+\mathbf{n}_{k},
$$

where $\mathbf{n}_{k}$ is the noise vector. Note that $\mathbf{v}_{0}=\frac{1}{M}[1,1, \cdots, 1]^{T}$. It can easily be shown that $\mathbf{v}_{k}^{H} \mathbf{v}_{0}=0, \forall k \neq 0$, thus compactly representing the fact that all the OFDM subcarriers are orthogonal to each other.

\section{B. Received signal for cases 2 and 3}

Let us consider an OFDM system with a frequency offset $f_{d} \mathrm{~Hz}$. The normalized CFO is then $\epsilon=f_{d} T$. The partial FFT outputs for such a scenario can be expressed as

$$
\begin{aligned}
& \mathbf{y}_{k}=\mathbf{D}_{\epsilon} \sum_{l=1}^{K} d_{l} H_{l} \mathbf{v}_{l-k}+\mathbf{n}_{k}, \epsilon=f_{d} T \\
& \mathbf{D}_{\epsilon}=\operatorname{diag}\left[e^{j 2 \pi \epsilon \frac{(2 m-1)}{2 M}}\right], m=1,2, \cdots, M .
\end{aligned}
$$

For OFDM signaling over a UWA channel with linearly varying path delays, we get

$$
\begin{aligned}
\mathbf{y}_{k} & =\sum_{l=1}^{K} d_{l} H_{l} \mathbf{D}_{\epsilon_{l}} \mathbf{v}_{l-k}+\mathbf{n}_{k}, \epsilon_{l}=a f_{l} T \\
\mathbf{D}_{\epsilon_{l}} & =\operatorname{diag}\left[e^{j 2 \pi \epsilon_{l} \frac{(2 m-1)}{2 M}}\right], m=1,2, \cdots, M .
\end{aligned}
$$

We note that for UWA systems with time scaling, though the frequency offset is different for each subcarrier, the difference between the frequency offsets of several adjacent subcarriers is negligible. This allows us to analyze the CFO scenario and interpret the obtained results for an underwater OFDM signal with time scaling.

\section{Combining Partial FFT Outputs}

If the partial FFT outputs are weighted before combining, a judicious selection of weights enables partial compensation for the time-varying channel response. The weighted combining is performed as follows. Let us define $\mathbf{w}_{k}=$ $\left[w_{k}(1), w_{k}(2), \cdots, w_{k}(M)\right]^{T}$ as the vector of the combiner weights for the $k^{\text {th }}$ subcarrier. The combining then yields a signal $x_{k}=\mathbf{w}_{k}^{H} \mathbf{y}_{k}$. If $\mathbf{w}_{k}=[1,1, \cdots, 1]^{T}, \forall k$, the situation is equivalent to performing a single, full-interval FFT. For the ideal case of a time-invariant channel (case 1), using the above weights yields $x_{k}=H_{k} d_{k}+n_{k}$ and symbol-by-symbol detection is optimal. When the channel is time-varying, the combiner weights are tailored to achieve the desired output in this form. In the next section, we compute the optimal combiner weights for the special cases of interest.

\section{Optimal COMBINING}

We first focus on deriving the minimum mean squared error (MMSE) optimal weights $\mathbf{w}_{k}^{o p t}$ for combining the partial FFT outputs assuming perfect knowledge of the time-varying channel. As we are interested in one-tap equalization of timevarying signals, it is assumed that only the partial FFT outputs for the $k^{t h}$ subcarrier are used to determine the symbol transmitted on this subcarrier. This assumption is qualified 
subsequently by showing that optimal combining significantly reduces the interference from adjacent carriers for several scenarios of interest. The optimal combiner weights are the solution to the MMSE optimization problem:

$$
\mathbf{w}_{k}^{o p t}=\min _{\mathbf{w}_{k}} E\left[\left|d_{k}-\mathbf{p}_{k}^{H} \mathbf{y}_{k}\right|^{2}\right],
$$

and can be evaluated as $E\left[\mathbf{y}_{k} \mathbf{y}_{k}^{H}\right] \mathbf{w}_{k}^{\text {opt }}=E\left[\mathbf{y}_{k} d_{k}^{*}\right]$. Substituting for $\mathbf{y}_{k}$ from (5) and evaluating the cross-correlation term, we get $\mathbf{R}_{\mathbf{y}_{k} d_{k}}=E\left[\mathbf{y}_{k} d_{k}^{*}\right]=\mathbf{H}_{k} \mathbf{v}_{0}$. The autocorrelation matrix of $\mathbf{y}_{k}, \mathbf{R}_{\mathbf{y}_{k}}$, can be derived as

$$
\mathbf{R}_{\mathbf{y}_{k}}=\sum_{l=1}^{K} \mathbf{H}_{l} \mathbf{v}_{l-k} \mathbf{v}_{l-k}^{H} \mathbf{H}_{l}^{H}+\frac{N_{0}}{M} \mathbf{I}_{M} .
$$

The optimal combiner weights can then be evaluated by substituting the cross-correlation vector and the auto-correlation matrix in the above MMSE solution in (8).

1) Time Invariant Channel: When the subcarrier frequency response coefficients $H_{k}(m)$ are independent of $m$, the channel is time invariant and the auto-correlation matrix can be simplified using $\mathbf{H}_{l}=H_{l} \operatorname{diag}([1,1, \ldots, 1])$. For a time invariant channel, it can be clearly seen that the auto-correlation matrix $\mathbf{R}_{\mathbf{y}_{k}}$ is a circulant matrix. Exploiting the fact that any circulant matrix is diagonalized by a DFT matrix, we get $\mathbf{R}_{\mathbf{y}_{k}}=\mathbf{F} \boldsymbol{\Lambda}_{k} \mathbf{F}^{H}$, where $\mathbf{F}$ is an $M \times M$ unitary DFT matrix and $\boldsymbol{\Lambda}_{k}$ is a diagonal matrix of the eigen values of $\mathbf{R}_{\mathbf{y}_{k}}$. The optimal combiner coefficients are then

$$
\mathbf{w}_{k}^{\text {opt }}=\frac{H_{k}}{M} \mathbf{F} \boldsymbol{\Lambda}_{k}^{-1} \mathbf{F}^{H} \mathbf{v}_{0}=\frac{H_{k}}{\lambda_{k}(1)} \mathbf{v}_{0},
$$

where, the equation is simplified by noting that $\mathbf{v}_{0}$ is also the first eigen vector of the DFT matrix and $\lambda_{k}(1)$ is the first diagonal element of $\boldsymbol{\Lambda}_{k}$ and is given as

$$
\lambda_{k}(1)=\sum_{m_{2}=1}^{M} \mathbf{R}_{\mathbf{y}_{k}}\left(1, m_{2}\right)=\frac{1}{M}\left(\left|H_{k}\right|^{2}+N_{0}\right),
$$

and the MMSE optimal combiner weights are then

$$
\mathbf{w}_{k}^{\text {opt }}=\frac{H_{k}}{\left|H_{k}\right|^{2}+N_{0}} M \mathbf{v}_{0}=\frac{H_{k}}{\left|H_{k}\right|^{2}+N_{0}}[1,1, \ldots, 1] .
$$

Thus, the optimal processing for a time-invariant channel would be to add all the partial FFT outputs to get a full FFT output and then equalize the computed data which is the standard OFDM processing for this scenario.

2) System with Carrier Frequency Offset: For an OFDM system with a normalized CFO $\epsilon=f_{d} T$, the partial FFT outputs are given from (6) as

$$
\mathbf{y}_{k}=\mathbf{D}_{\epsilon} \sum_{l=1}^{K} d_{l} H_{l} \mathbf{v}_{l-k}+\mathbf{n}_{k} .
$$

Evaluating the auto-correlation and cross-correlation, we get

$$
\begin{aligned}
\mathbf{R}_{\mathbf{y}_{k}} & =\mathbf{D}_{\epsilon} \sum_{l=1}^{K}\left|H_{l}\right|^{2} \mathbf{v}_{l-k} \mathbf{v}_{l-k}^{H} \mathbf{D}_{\epsilon}^{H}+\frac{N_{0}}{M} \mathbf{I}_{M} \\
\mathbf{R}_{\mathbf{y}_{k} d_{k}} & =\mathbf{D}_{\epsilon} H_{k} \mathbf{v}_{0} .
\end{aligned}
$$

Noting the fact that $\mathbf{D}_{\epsilon} \mathbf{D}_{\epsilon}^{H}=\mathbf{I}_{M}$ and simplifying as done in the case of a time-invariant channel, the optimal combiner weights in this scenario are derived as

$$
\mathbf{w}_{k}^{\text {opt }}=\mathbf{D}_{\epsilon} \frac{H_{k}}{\left|H_{k}\right|^{2}+N_{0}} M \mathbf{v}_{0} .
$$

The structure of the optimal combiner coefficients suggests that the optimal receiver first compensates for the approximated phase distortion due to the frequency offset and perform the same processing as in the time-invariant case.

3) Wideband system with time scaling due to Doppler distortion: As mentioned earlier, for OFDM signaling on channels with time scaling, we observe that each subcarrier is shifted by a slightly different frequency and thus this scenario can be considered as a generalization of OFDM with frequency offset. The outputs of the partial FFT blocks are given from (7) as

$$
\mathbf{y}_{k}=\sum_{l=1}^{K} d_{l} H_{l} \mathbf{D}_{\epsilon_{l}} \mathbf{v}_{l-k}+\mathbf{n}_{k}, \epsilon_{l}=a f_{l} T
$$

Evaluating the auto-correlation and the cross-correlation terms as in the previous case, we get

$$
\begin{aligned}
\mathbf{R}_{\mathbf{y}_{k}} & =\sum_{l=1}^{K}\left|H_{l}\right|^{2} \mathbf{D}_{\epsilon_{l}} \mathbf{v}_{l-k} \mathbf{v}_{l-k}^{H} \mathbf{D}_{\epsilon_{l}}^{H}+\frac{N_{0}}{M} \mathbf{I}_{M} \\
\mathbf{R}_{\mathbf{y}_{k} d_{k}} & =\mathbf{D}_{\epsilon_{k}} H_{k} \mathbf{v}_{0} .
\end{aligned}
$$

Evaluating the terms in the auto-correlation matrix, we get

$$
\begin{aligned}
\mathbf{R}_{\mathbf{y}_{k}}\left(m_{1}, m_{2}\right)= & \frac{1}{M^{2}} \sum_{l=1}^{K}\left|H_{l}\right|^{2} e^{j 2 \pi\left(l-k+\epsilon_{l}\right) \frac{\left(m_{1}-m_{2}\right)}{M}} \times \\
& \operatorname{sinc}^{2}\left(\frac{\pi(l-k)}{M}\right)+N_{0} / M
\end{aligned}
$$

From the above equation, we observe that the auto-correlation matrix is no longer a circulant matrix and hence a simple and intuitive solution to the optimal weights $w_{k}^{o p t}$ may be intractable. However, $\mathbf{R}_{\mathbf{y}_{k}}$ is Toeplitz Hermitian matrix and can be approximated with a circulant matrix for large $M$ [14]. Using the circulant approximation for the auto-correlation matrix of the data, we get

$$
\mathbf{w}_{k}^{o p t} \approx \mathbf{D}_{\epsilon_{k}} \frac{H_{k}}{\lambda_{k}(1)} M \mathbf{v}_{0},
$$

where $\lambda_{k}(1)$ is the first eigen value of the approximated circulant matrix. The value of $\lambda_{k}(1)$ is dominated by the term $\left|H_{k}\right|^{2}+N_{0}$ but includes several other terms corresponding to the channel gains of other subcarriers. We note that for large values of $M$, the optimal strategy reduces to compensating the phase distortion on each partial interval and then equalizing the received data as in the case with CFO. However, we note that the matrix $\mathbf{D}_{\epsilon_{k}}$ is now dependent on the subcarrier.

\section{AnAlysis of Estimation Algorithms}

We now analyze the performance of the proposed weight estimation algorithms proposed in [6] for the CFO scenario. The analysis for this scenario gives valuable insight into the 
effectiveness of partial FFT demodulation for compensating time scaling distortion.

Let us first consider model based estimation assuming that the model parameters are estimated correctly. Assuming that the channel parameters are deterministic, the signal at the $k^{t h}$ subcarrier is $\left[\sum_{m=1}^{M} e^{-j 2 \pi \epsilon \frac{2 m-1}{2 M}} y_{k}(m)\right]$. Substituting for the exact expression for $y_{k}(m)$ from (6), we get the signal power $\mathrm{SP}_{k}=\left|H_{k}\right|^{2} \operatorname{sinc}^{2}\left(\frac{\pi \epsilon}{M}\right)$. The total power of the noise and interference for the $k^{t h}$ subcarrier can be similarly calculated as

$$
\mathrm{IP}_{k}=N_{0}+\sum_{l=1, l \neq k}^{K}\left|H_{l}\right|^{2} \operatorname{sinc}^{2}\left(\frac{\pi(l-k+\epsilon)}{M}\right) \times C_{l-k}(M),
$$

where the interference term $C_{l-k}(M)=$ $\left|\sum_{m=1}^{M} \frac{1}{M} e^{j 2 \pi(l-k) \frac{(2 m-1)}{2 M}}\right|^{2}$ is non-zero only for subcarriers for which $(l-k)$ is a multiple of $M$. Thus, for a time-invariant channel with frequency offset, the partial FFT followed by optimal combining fully eliminates the interference from all the subcarriers which are not at multiples of $M$ away from the subcarrier of interest. Thus, even for moderate values of $M$, there is no interference from adjacent subcarriers and thus results in a significant improvement in signal to interference ratio (SIR). The SIR given by $\mathrm{SP}_{k} / \mathrm{IP}_{k}$ is then

$$
\operatorname{SIR}_{k}^{*}(M)=\frac{\left|H_{k}\right|^{2} \operatorname{sinc}^{2}\left(\frac{\pi \epsilon}{M}\right)}{N_{0}+\sum_{l=1, l \neq k, l-k=p M}^{K}\left|H_{l}\right|^{2} \operatorname{sinc}^{2}\left(\frac{\pi(p M+\epsilon)}{M}\right)} .
$$

Clearly, the signal power is monotonically increasing in $M$ and the number of zero terms in the interference expression increase for increasing $M$. Thus, for a fixed channel frequency response, the SIR is an increasing function of $M$. However, as $M$ increases, the number of partial FFT's to be performed also increases. The practical value of $M$ to be used is then only limited by the computational cost of the partial FFT.

\section{A. Analysis of Recursive Weight Estimation}

The recursive weight estimation algorithm runs adaptively over the subcarriers and its performance is a function of the rate of variation of the channel frequency response and the number of intervals into which the OFDM symbol is divided. Intuitively, when $M$ increases from a very small value the performance of recursive estimation would improve as the distortion process is better modeled for larger $M$. However, upon increasing $M$ further, the number of weights to be estimated increases and the accuracy of estimating the RLS covariance matrix decreases and thus we observe a performance degradation for larger values of $M$ [6]. To quantify the tradeoff between better modeling the distortion and the accuracy of the RLS parameters, we consider the analysis of the RLS algorithm under steady state conditions.

Due to the finite number of observations and the frequency selectiveness of the time-varying channel, the estimated input covariance matrix $\hat{\mathbf{R}}_{\mathbf{y}_{k}}$ is highly time-varying and the analysis of the general problem may not be very tractable. To provide us intuition into the trade-off and to determine an optimal $M^{*}$ for practical implementations, we consider the analysis under several simplifying assumptions. Since the observed trade-off is due to the adaptive RLS algorithm, we focus our analysis on the performance of RLS by assuming a flat fading channel with gain $h_{0}$ and that the transmitted symbols are accurately known at the receiver. This assumption allows us to approximate the data covariance matrix $\mathbf{R}_{\mathbf{y}_{k}}$ as subcarrier-invariant. An analysis of the expression

$$
\mathbf{R}=E_{d}\left|h_{0}\right|^{2}\left[\sum_{l=1}^{K} \mathbf{v}_{l-k} \mathbf{v}_{l-k}^{H}\right]+\frac{N_{0}}{M} \mathbf{I}_{M},
$$

shows that the approximation is accurate for most of the subcarriers except for the ones at the edges of the OFDM symbol. The parameter $E_{d}$ is the average energy of the transmitted constellation and is modeled explicitly in this section. Considering separately the signal and interference, we can write $\mathbf{R}=E_{d}\left|h_{0}\right|^{2} \mathbf{v}_{0} \mathbf{v}_{0}^{H}+\mathbf{R}_{I}$, where $\mathbf{R}_{I}$ is the covariance matrix of just the interfering terms with the subcarrier of interest. Using the matrix-inversion lemma it can be shown that the covariance matrix of the data and the interference are related as

$$
\frac{1}{\mathbf{v}_{0}^{H} \mathbf{R}^{-1} \mathbf{v}_{0}}=E_{d}\left|h_{0}\right|^{2}+\frac{1}{\mathbf{v}_{0}^{H} \mathbf{R}_{I}^{-1} \mathbf{v}_{0}} .
$$

As we consider an exponentially weighted RLS algorithm [6], the optimization function for the combiner weights is given as

$$
\sum_{i=1}^{n} \lambda^{n-i}\left|\sqrt{E_{d}} d_{i}-\mathbf{w}^{H}(n) \mathbf{y}_{i}\right|^{2}
$$

The RLS adaptation rule in this case is given as

$$
e_{k}=\sqrt{E_{d}} d_{k}-\mathbf{w}_{k-1}^{H} \mathbf{y}_{k}, \mathbf{w}_{k}^{H}=\mathbf{w}_{k-1}^{H}+e_{k} g_{k},
$$

where $e_{k}$ is the prediction error at subcarrier $k$ and $g_{k}$ is the gain vector from the RLS update. Using results from RLS convergence theory, the mean weight vector $\mathbf{w}_{k}^{H}$ converges to the optimal linear MMSE solution $\mathbf{w}^{\text {opt }}=E_{d}\left|h_{0}\right|^{2} \mathbf{R}^{-1} \mathbf{v}_{0}$. It can be shown that the mean squared error (MSE) of the estimation error converges to $\left|e_{k}\right|^{2} \rightarrow e^{*}+e_{e x}(\infty)$, where $e^{*}$ is the mean-square error of the optimum filter $\mathbf{w}^{o p t}$, given by

$$
\begin{aligned}
e^{*} & =E\left[\left|\sqrt{E_{d}} d_{k}-\mathbf{w}^{\text {opt }} \mathbf{y}_{k}\right|^{2}\right] \\
& =E_{d}\left|h_{0}\right|^{2}\left(1-E_{d}\left|h_{0}\right|^{2} \mathbf{v}_{0}^{H} \mathbf{R}^{-1} \mathbf{v}_{0}\right) \\
& =\frac{E_{d}\left|h_{0}\right|^{2}}{1+E_{d}\left|h_{0}\right|^{2} \mathbf{v}_{0}^{H} \mathbf{R}_{I}^{-1} \mathbf{v}_{0}}=\frac{E_{d}\left|h_{0}\right|^{2}}{1+\mathrm{SIR}^{*}},
\end{aligned}
$$

where $\mathrm{SIR}^{*}=E_{d}\left|h_{0}\right|^{2} \mathbf{v}_{0}^{H} \mathbf{R}_{I}^{-1} \mathbf{v}_{0}$ [12]. The steady-state excess mean-square error is given by $e_{e x}(\infty)=\frac{1-\lambda}{1+\lambda} M e^{*}=\eta e^{*}$ [13]. Now, under the assumption of known data symbols, the steady-state SIR output of the RLS update is computed. The second moment and the mean of the output at steady state are

$$
\begin{aligned}
E\left[\left|\mathbf{w}_{k-1}^{H} \mathbf{y}_{k}\right|^{2}\right] & =e_{k}+2 \sqrt{E_{d}} d_{k} E\left[\mathbf{w}_{k-1}^{H} \mathbf{y}_{k}\right]-E_{d}\left|h_{0}\right|^{2} \\
& \rightarrow e_{e x}(\infty)-E_{d}\left|h_{0}\right|^{2}\left(1-2\left(\mathbf{w}^{o p t}\right)^{H} \mathbf{v}_{0}\right)
\end{aligned}
$$

and

$$
E\left[\mathbf{w}_{k-1}^{H} \mathbf{y}_{k}\right] \rightarrow \sqrt{E_{d}} d_{k}\left(\mathbf{w}^{o p t}\right)^{H} \mathbf{v}_{0}=\sqrt{E_{d}} h_{0} d_{k} \frac{S I R^{*}}{1+S I R^{*}} .
$$




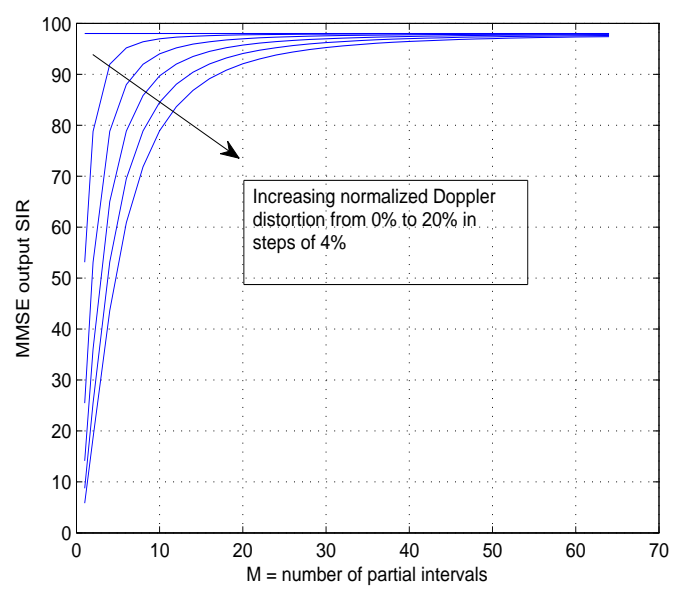

Fig. 1. Optimal SIR as a function of $M$ averaged over a Rayleigh fading channel, $\mathrm{SNR}=20 \mathrm{~dB}$

From the mean and the second moment of the output, the variance of the estimates can be computed as

$$
\operatorname{var}\left[\mathbf{w}_{k-1}^{H} \mathbf{y}_{k}\right] \rightarrow \frac{(1+\eta) S I R^{*}+\eta}{\left(1+S I R^{*}\right)^{2}} E_{d} .
$$

The steady-state SIR can then be derived as

$$
S I R^{\infty}=\lim _{n \rightarrow \infty} \frac{\left|E\left[\mathbf{w}_{k-1}^{H} \mathbf{y}_{k}\right]\right|^{2}}{\operatorname{var}\left[\mathbf{w}_{k-1}^{H} \mathbf{y}_{k}\right]}=\frac{S I R^{*}}{(1+\eta)+\eta /\left(S I R^{*}\right)}
$$

The above equation gives the steady-state SIR for the recursive estimation algorithm to characterize the performance tradeoff due to the RLS algorithm. We plot the steady state SIR under the flat fading channel and constant data covariance matrix assumptions for both the recursive and the model based weight estimation algorithms. Figs. 1 and 2 show the plots of SIR (averaged over the Rayleigh fading channel gain) as a function of the number of partial FFT intervals respectively. As predicted by the analysis in this paper and as seen in the numerical simulations in [6], the model based estimator has monotonically improving performance as a function of $M$. For recursive weight estimation, the SIR curve peaks at a certain value of $M$ depending on the normalized CFO due to better distortion modeling and degrades later due to the adaptation inaccuracies of the RLS parameters.

\section{Conclusions}

Time scaling due to Doppler distortion in underwater acoustic communications using OFDM signaling causes different frequency offsets for each subcarrier and results in significant inter-carrier interference if not compensated for. We proposed a new technique partial FFT demodulation in [6] and presented two algorithms for time scale compensation: model based weight estimation and recursive weight estimation,. In this paper, we presented an analysis of the performance of the two algorithms and showed that the performance of model based estimation increases monotonically with the number of partial FFT intervals $M$. For recursive weight estimation, using an approximated analysis, the trade-off between modeling

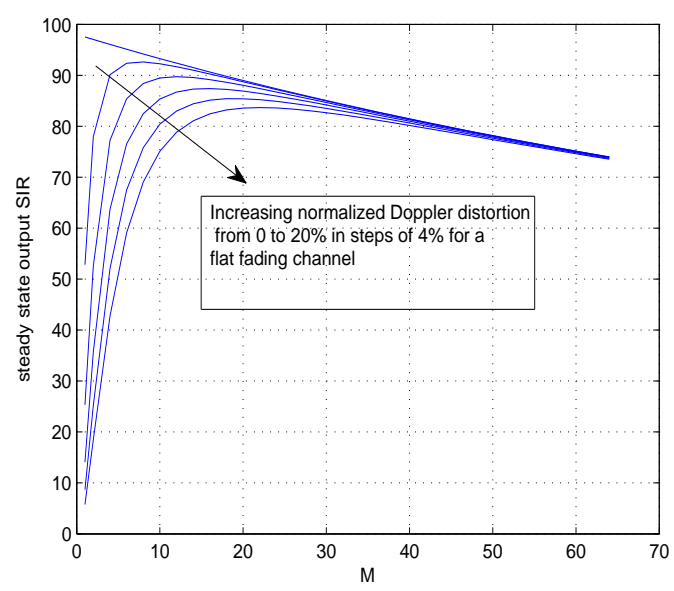

Fig. 2. Steady state SIR after RLS convergence as a function of $M$ averaged over a Rayleigh fading channel, SNR $=20 \mathrm{~dB}$

and estimation errors is characterized and it is shown that performance improves initially with increasing $M$ due to better distortion modeling and then decreases as $M$ increases further due to inaccurate estimation of key parameters.

\section{REFERENCES}

[1] M. Stojanovic, "Underwater acoustic communications: Design considerations on the physical layer," in Fifth Annual Conference on Wireless on Demand Network Systems and Services, WONS, Jan 2008, pp. 1-10.

[2] - "MIMO OFDM over underwater acoustic channels," in 43rd Asilomar Conference on Signals, Systems and Computers, Nov 2009.

[3] B. Li, S. Zhou, M. Stojanovic, L. Freitag, and P. Willet, "Multicarrier communication over underwater acoustic channels with nonuniform Doppler shifts," IEEE Journal of Oceanic Engineering, vol. 33, no. 2, April 2008.

[4] S. Yerramalli, M. Stojanovic, and U. Mitra, "Partial FFT demodulation: A detection method for Doppler distorted OFDM systems," in Intl. Workshop on Sig. Proc. Advances in Wireless Comm., (SPAWC) 2010.

[5] B.-C. Kim and I.-T. Lu, "Parameter study of underwater communication system," in OCEANS 2000 MTS/IEEE Conference and Exhibition, vol. 2, 2000, pp. 1251-1255.

[6] S. Yerramalli, M. Stojanovic, and U. Mitra, "Data detection algorithms for Doppler distorted OFDM systems," in WUWNet '10: Proc. of the 5th ACM international workshop on Underwater networks, Nov 2010.

[7] A. B. Salberg and A. Swami, "Doppler and frequency offset synchronization in wideband OFDM," IEEE Transactions on Wireless Communications, vol. 4, no. 6, pp. 2870-2881, Nov 2006.

[8] M. Stojanovic, "Adaptive channel estimation for underwater acoustic MIMO OFDM systems," in Proceedings of IEEE DSP/SPE Workshop, Jan 2009.

[9] — , "A method for differentially coherent detection of OFDM signals on Doppler distorted channels," in Proc. 6-th IEEE Sensor Array and Multichannel Signal Processing Workshop (SAM), Oct 2010.

[10] K. Tu, D. Fertonani, T. M. Duman, and P. Hursky, "Mitigation of intercarrier interference in OFDM systems over underwater acoustic channels," in Proceedings of IEEE Oceans Conference, May 2009.

[11] S.-J. Hwang and P. Schniter, "Efficient communication over highly spread underwater acoustic channels," in WuWNet '07: Proceedings of the second workshop on Underwater networks, 2007, pp. 11-18.

[12] W. Chen and U. Mitra, "An improved blind adaptive MMSE receiver for fast fading DS-CDMA channels," IEEE Journal on Selected Areas in Communications, vol. 19, no. 8, Aug 2001.

[13] H. V. Poor and X. Wang, "Code-aided interference supression for DS/CDMA communications-part II: Parallel blind adaptive implementations," IEEE Transactions on Communcations, vol. 45, no. 9, 1997.

[14] R. M. Gray, Toeplitz and Circulant matrices: A review. Now Publishers. 